\title{
Variable selection methods for multiple regressions influence the parsimony of risk prediction models for cardiac surgery
}

\author{
Md Nazmul Karim, MBBS, M Clin Epi, ${ }^{a}$ Christopher M. Reid, PhD, ${ }^{\text {a,b }}$ Lavinia Tran, PhD, ${ }^{\mathrm{a}}$ \\ Andrew Cochrane, MD, FRCS, ${ }^{\mathrm{c}}$ and Baki Billah, $\mathrm{PhD}^{\mathrm{a}}$
}

\begin{abstract}
Objective: To compare the impact of different variable selection methods in multiple regression to develop a parsimonious model for predicting postoperative outcomes of patients undergoing cardiac surgery.

Methods: Data from 84,135 patients in the Australian and New Zealand Society of Cardiac and Thoracic Surgeons registry between 2001 and 2014 were analyzed. Primary outcome was 30-day-mortality. Mixed-effect logistic regressions were used to build the model. Missing values were imputed by the use of multiple imputations. The following 5 variable selection methods were compared: bootstrap receiver-operative characteristic (ROC), bootstrap Akaike information criteria, bootstrap Bayesian information criteria, and stepwise forward and stepwise backward methods. The final model's prediction performance was evaluated by the use of Frank Harrell's calibration curve and using a multifold crossvalidation approach.
\end{abstract}

Results: Stepwise forward and backward methods selected same set of 21 variables into the model with the area under the ROC (AUC) of 0.8490. The bootstrap ROC method selected 13 variables with AUC of 0.8450. Bootstrap Bayesian information criteria and Akaike information criteria respectively selected 16 (AUC: 0.8470) and 23 (AUC: 0.8491) variables. Bootstrap ROC model was selected as the final model which showed very good discrimination and calibration power.

Conclusions: Clinical suitability in terms of parsimony and prediction performance can be achieved substantially by using the bootstrap ROC method for the development of risk prediction models. (J Thorac Cardiovasc Surg 2017;153:1128-35)

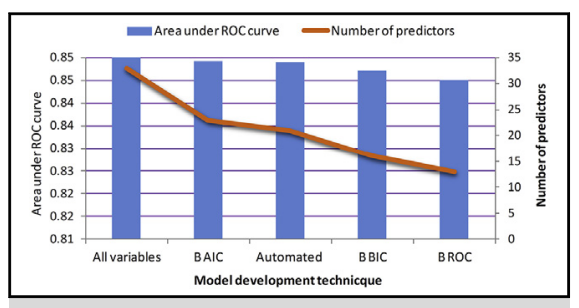

The bootstrap receiver operating characteristic method provides parsimony of risk prediction model.

\section{Central Message}

The balance between parsimony and performance can be improved using the bootstrap method with receiver operating characteristics for developing risk prediction models.

\section{Perspective}

The trade-off between parsimony and performance is a major challenge in risk prediction modeling. Different approaches to variable selection may be an avenue for improving the parsimony of a cardiac surgical risk prediction model. This work compared the clinical suitability of models generated by the use of different variable selection methods with regard to parsimony and performance to predict 30-day mortality.

See Editorial Commentary page 1136.
In the past decades, the field of cardiac surgery has made significant progress in the development of risk prediction models to enable outcome prediction and clinical quality monitoring. National cardiac surgical registries have been established in many countries, and many have developed

\footnotetext{
From the a School of Public Health and Preventive Medicine, Department of Epidemiology and Preventive Medicine, Monash University, Melbourne; ${ }^{\mathrm{b}}$ School of Public Health, Department of Health Policy and Management, Curtin University, Western Australia; and ${ }^{c}$ Department of Cardiothoracic Surgery, Monash Medical Centre, Clayton, Victoria, Australia.

Received for publication Dec 1, 2015; revisions received Oct 24, 2016; accepted for publication Nov 14, 2016; available ahead of print Dec 19, 2016.

Address for reprints: Baki Billah, PhD, Department of Epidemiology and Preventive Medicine, Faculty of Medicine Nursing and Health Science, Monash University, The Alfred Centre, (level 6), 99 Commercial Rd, Melbourne, VIC 3004, Australia (E-mail: baki.billah@monash.edu).

$0022-5223 / \$ 36.00$

Copyright (c) 2016 by The American Association for Thoracic Surgery

http://dx.doi.org/10.1016/j.jtcvs.2016.11.028
}

risk prediction models suitable for local populations. ${ }^{1-3}$ The aim of these models was to provide an estimate of postoperative mortality risk based on preoperative risk factors.

In risk prediction modeling of cardiac surgery, outcomes data are adjusted for preoperative risk factors. When so many variables are included in the model, however, the accuracy along with parsimony of the model may be compromised. ${ }^{4}$ The principle of parsimony states that

Scanning this QR code will take you to supplemental tables for this article. 


\section{Abbreviations and Acronyms \\ AIC $=$ Akaike information criteria \\ ANZSCTS $=$ Australian and New Zealand Society \\ of Cardiac and Thoracic Surgeons \\ Registry \\ AUC = Area under ROC curve \\ BIC $=$ Bayesian information criteria \\ $\mathrm{CI}=$ confidence interval \\ ROC $=$ receiver operating characteristic}

simpler explanations are preferred over more complex explanations. ${ }^{5}$ A parsimonious model is computationally simpler for the clinician to implement in day-to-day practice. ${ }^{5} \mathrm{~A}$ smaller number of variables in a risk prediction model makes the model simpler to use. Furthermore, when many variables are included in the model, the burden of clinical staff is increased considerably in regard to data collection, which can compromise data quality as well. The prevalence of cases with missing data also may increase with increasing number of variables in the model. If a simple model can explain a phenomenon with a similar level of accuracy compared with a complex model, the former model should be preferred on the ground of parsimony, unless the complex model outperforms the former. ${ }^{6,7}$

Several methodologic approaches are currently in practice for identifying the predictor variables for risk prediction models. These methods excel in different tasks and have their inherent limitations. Automated variable selection methods are used widely for model development. ${ }^{8}$ Automated variable selection, forward selection, and backward elimination are very easy to use and available in most statistical software packages. Although popularly used, a number of concerns have been identified with the application of these methods in the field of risk prediction modeling. ${ }^{9}$

Bootstrap resampling is another approach used for variable selection in risk prediction modeling. ${ }^{10-12}$ Usually bootstrap resampling is used in conjunction with criteria like Akaike information criteria (AIC) ${ }^{13}$ Bayesian information criteria (BIC), ${ }^{14}$ and receiver operating characteristics (ROCs) ${ }^{15}$ to develop models; however, the question of whether developing models using bootstrapping method in combination with these criteria improves the parsimony of a risk prediction model remains unclear. Therefore, comparison of different methodologic approaches for variable selection may be an avenue for improving the parsimony of risk prediction models.

The aim of this paper was to compare the clinical suitability of risk prediction models generated by the use of different variable selection methods with regard to their parsimony and performance to predict postoperative outcomes of cardiac surgery patients.

\section{METHODS}

\section{Australian and New Zealand Society of Cardiac and Thoracic Surgeons Registry (ANZSCTS) Database}

The ANZSCTS collects information on adult patients undergoing cardiac surgery in 28 hospitals across Australia. The database collects 287 preoperative, intraoperative, and postoperative variables via internationally standardized data definitions (Table E1). ${ }^{3}$ The data collection and its audit methods have been discussed elsewhere. ${ }^{3,16}$ Current analysis included information of 84,135 patients who underwent cardiac surgery between 2001 and 2014. Of them, 63,523 patient records between 2001 and 2012 were used for model development and 20,625 patient records between 2013 and 2014 were used for validation. The primary outcome variable for this study was 30-day mortality. ${ }^{17}$ The variable "New York Heart Association classification" (5.28\%) had the highest missing data, followed by "ejection fraction" (3.07\%), "procedure type" $(1.24 \%)$, and "number of diseased vessels" (1.11\%). The remaining predictors had $<1 \%$ missing observations (Table E2).

\section{Plausible Predictor Identification and Model Development Methods}

Of 287 variables (data field) in the ANZSCTS registry, 101 were preoperative variables. These preoperative variables included administrative data and several stems of variables. On the basis of extensive literature review on cardiac surgery risk prediction models and clinical judgment, a total of 52 variables were identified primarily as preoperative risk factors. With the use of bivariate mixed-effect logistic regression, 33 variables finally were identified as plausible risk factors for 30-day mortality.

Multiple imputation of missing value was performed with the imputation by chained equations method along with multivariable mixed-effect logistic regression. Data were "filled in" with imputed values generated by the use of a specified regression model. The process was repeated 3 times to generate 3 completed datasets. Studies have shown that repeating imputation 3 times is sufficient for data with less missing data $(<20 \%) .^{18}$ The mixed-effect logistic regression was fitted separately on each of the imputed dataset. The results were then pooled into an aggregated estimate following Rubin's rule ${ }^{19}$ through the MIM estimation command option in Stata 14. ${ }^{20}$ Multivariable mixed-effect logistic regression was used for the risk prediction. The multilevel modeling accounts for potential between-hospital variations. $^{21}$ The first order interaction effect between clinically relevant risk factors was investigated.

Nonlinearity of continuous predictors (age, body mass index, and estimated glomerular filtration rate) was addressed by fitting fractional polynomial in the mixed-effect logistic regression model. ${ }^{22,23}$ Sensitivity analysis was performed for nonlinear term in the model. Little improvement in discrimination and calibration was seen with inclusion of nonlinear terms in the model; hence, linear terms of the continuous variables were kept in the final model to keep the model simple and user friendly. The following 5 variable selection methods were compared: (1) bootstrap along with ROC, (2) bootstrap along with AIC, (3) bootstrap along with BIC, (4) stepwise forward selection method, and (5) stepwise backward elimination method. A model with all 33 variables also was developed.

\section{Bootstrap Model Selection}

A bootstrap sample of the same size of the original sample was drawn from each of the 3 imputed datasets. The 33 plausible risk factors were entered into the mixed-effect logistic regression and were applied to the bootstrap sample to test the significance of the variables. A variable with 
$P$ value of less than or equal to .05 was considered as significant. The process was repeated 1000 times, and the percentage of times that each variable appeared as significant in 1000 bootstraps (bootstrap coverage) in the imputed datasets was recorded. Bootstrap coverage of each predictor in 3 imputed dataset was averaged to generate an overall coverage of individual predictors. The predictors were then ranked depending on the overall bootstraps coverage. ${ }^{24}$

Fourteen plausible models were developed from variables that were significant in at least $50 \%$ of the bootstrap samples. ${ }^{25}$ Model 1 comprised 10 predictors that appeared as significant in $100 \%$ of bootstrap samples. Then, 13 subsequent models were generated through adding one variable at a time of decreasing rank according to the bootstrap coverage. The area under ROC curve (AUC), AIC, and BIC values were calculated for all of these 14 models. Both AIC and BIC methods select the model with their lowest values. Bootstrap ROC method selects the model with the greatest AUC value.

\section{Automated Model Selection}

Variables in the forward and backward logistic regression were selected for the imputed data as suggested by Wood and colleagues. ${ }^{24}$ The selected variables were then entered into the mixed-effect logistic regression and the model was estimated with multiple imputation, MIM estimation command option, in Stata $14 .{ }^{20}$ The AUC, AIC, and BIC values were calculated for final automated model.

\section{Discrimination and Calibration of the Models}

Selected models' prediction performance was evaluated by the use of discrimination and calibration powers. Model discrimination was evaluated with AUC. The calibration was evaluated with a decile-decile plot of the observed and predicted 30-day mortality. To calculate the calibration intercept and slope parameters, a linear regression model was fitted with the deciles of observed outcome as the dependent variable and the deciles of predicted outcome as the independent variable. Calibration of final model was also assessed using the method suggested by Frank Harrell with Regression Modeling Strategies (RMS) package in $\mathrm{R}$ statistical software?

\section{External Validation of the Final Model}

External validation of the final model was done on the validation dataset comprising patients treated during 2013 to 2014.

\section{Ethical Approval}

The institutional review board of each participating hospital approved the use of these databases for research (Alfred HREC:262/09). The ANZSCTS database registry approved collection of patient data via optout consent approach (MUHREC:CF08/0322-2008000065). The study received Ethical approval from Monash University, Standing Committee on Ethics in Research Involving Humans (SCERH) (MUHREC:CF14/ 1117-2014000476).

\section{RESULTS}

A total of 33 preoperative variables were identified as potential predictors (Tables E3 and E4). There was no firstorder interaction effect between these predictors. The percentage of times each of these candidate variables appeared as significant in the multiple mixed-effect logistic regression models in 1000 bootstraps were summarized in Table 1.

Figure 1 shows the AUC of 14 bootstrap models plotted against number of variables based on bootstrap coverage increment. The model with 10 predictors those appeared
TABLE 1. Summary of appearance of variables as independent predictors of mortality following cardiac surgery in bootstrap resampling

\begin{tabular}{llr}
\hline No. & \multicolumn{1}{c}{ Predictors } & $\% *$ \\
\hline 1 & Age & 100.0 \\
2 & Sex & 100.0 \\
3 & Previous cardiac surgery & 100.0 \\
4 & Peripheral vascular disease & 100.0 \\
5 & Stroke & 100.0 \\
6 & Glomerular filtration rate & 100.0 \\
7 & Infective endocarditis & 100.0 \\
8 & Urgency of procedure & 100.0 \\
9 & Type of procedure & 100.0 \\
10 & Ejection fraction & 100.0 \\
11 & Inotropic medication & 99.93 \\
12 & Angina & 99.83 \\
13 & NYHA class & 99.57 \\
14 & Dialysis & 90.17 \\
15 & Congestive heart failure & 89.80 \\
16 & Shock & 87.13 \\
17 & Myocardial infarction & 86.27 \\
18 & Resuscitation & 86.80 \\
19 & Arrhythmia & 81.00 \\
20 & Left main disease $<50 \%$ & 70.77 \\
21 & Hypertension & 68.00 \\
22 & Respiratory disease & 56.00 \\
23 & Hypercholesterolemia & 55.63 \\
24 & Cerebrovascular disease & 30.00 \\
25 & Steroid use & 31.17 \\
26 & Pacemaker in situ & 27.27 \\
27 & Body mass index & 21.20 \\
28 & Diabetes & 19.97 \\
29 & Number of disease vessel & 14.10 \\
30 & Immunosuppressant use & 11.50 \\
31 & Family history of heart disease & 12.87 \\
32 & Anticoagulant use & 7.53 \\
33 & Intra venous nitrate use & \\
resampling. & & York Heart Association. *Percentage of times of appearance in \\
& & \\
\hline & &
\end{tabular}

as significant in $100 \%$ of the bootstrap samples had an AUC of 0.8392 . With each addition of predictors, the AUC increased steeply until the model with 13 predictors (AUC 0.8450) appeared as significant in at least $99.6 \%$ of the samples and apparently reached a plateau (Figure 1). The addition of a further variable into the model didn't result in a steep increase in AUC (0.8454), which also corresponded to the sharp decrease of bootstrap coverage ( $99.6 \%$ with 13 variables vs $90.2 \%$ with 14 variables). Hence, the model with 13 predictors was selected as the final bootstrap ROC model. AIC and BIC values for all of the 14 competing bootstrap models are presented in Figure 2. The model with 23 variables had the lowest AIC $(12,326.5)$ and the model with 16 variables had the lowest BIC $(12,584.8)$. Hence, the model with 23 variables and the model with 16 variables were selected as bootstrap 


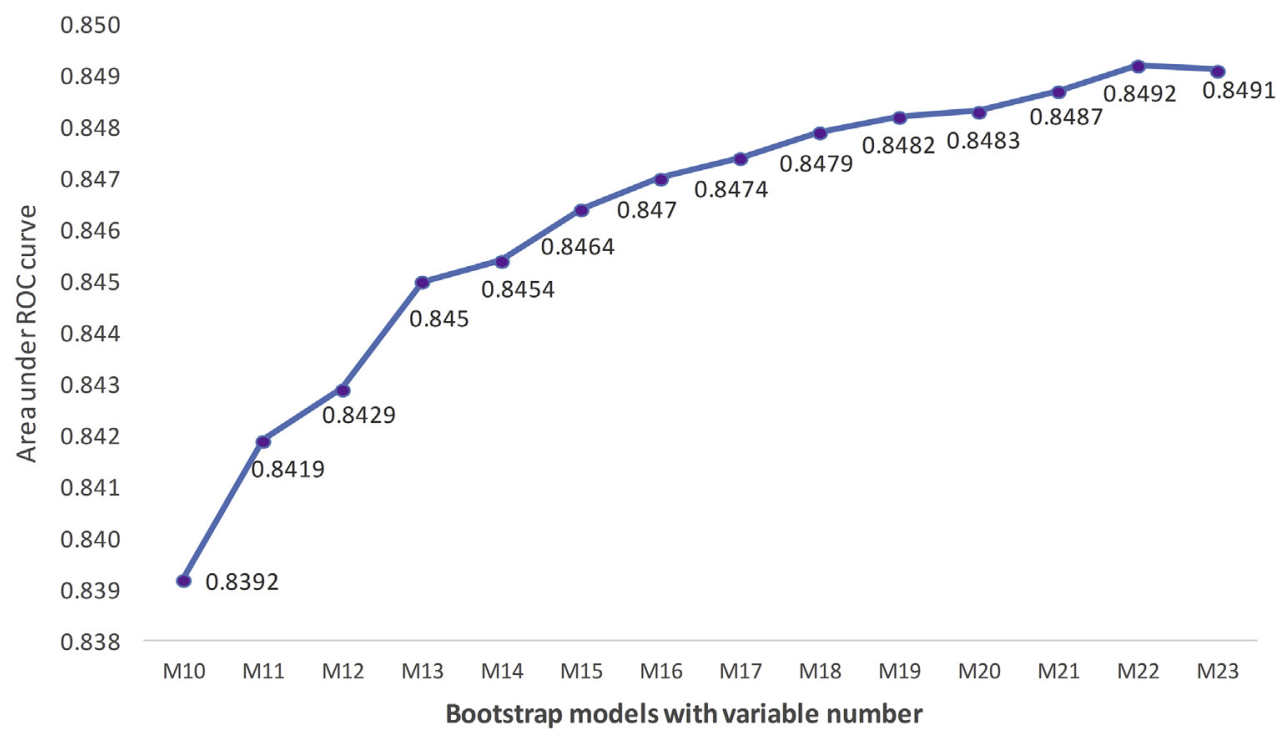

FIGURE 1. AUC gradient with increasing number of variables based on bootstrap coverage increment. AUC, Area under the ROC curve.

AIC and BIC model, respectively. The AUC values for bootstrap AIC and BIC models were, respectively, 0.8470 and 0.8491 .

The stepwise forward selection and backward elimination methods selected same set of 21 variables into the model with AUC of 0.8490 . The model with all 33 predictors had an AUC of 0.8504 .

The observed 30-day mortality in development sample was $2.693 \%$.The predicted mortality for all variable model was $2.524 \%$ (95\% confidence interval [CI], 2.481-2.567) and for the automated model was $2.524(95 \%$ CI, 2.481-2.567). The predicted risk for the bootstrap AIC, BIC, and ROC models were $2.529 \% \quad(95 \%$ CI,
$2.487-2.572), \quad 2.584 \% \quad(95 \% \quad$ CI, $2.540-2.627)$, and $2.574 \%$ (95\% CI, 2.531-2.616), respectively.

Figure 3 shows the calibration of the competing 5 models and the final model in validation sample. Bootstrap BIC, AIC, and ROC models showed calibration slopes of 1.0128, 1.0219 , and 1.0191, respectively. The automated and all variable models had calibration slopes of 1.0271 and 1.0133 , respectively. All of these models have intercepts close to zero.

Based on the number of variables and discrimination and calibration, the bootstrap ROC model with 13 variables was chosen as the final model (Table 2). The overfitting-corrected loess nonparametric calibration curve demonstrated excellent calibration for the bootstrap ROC

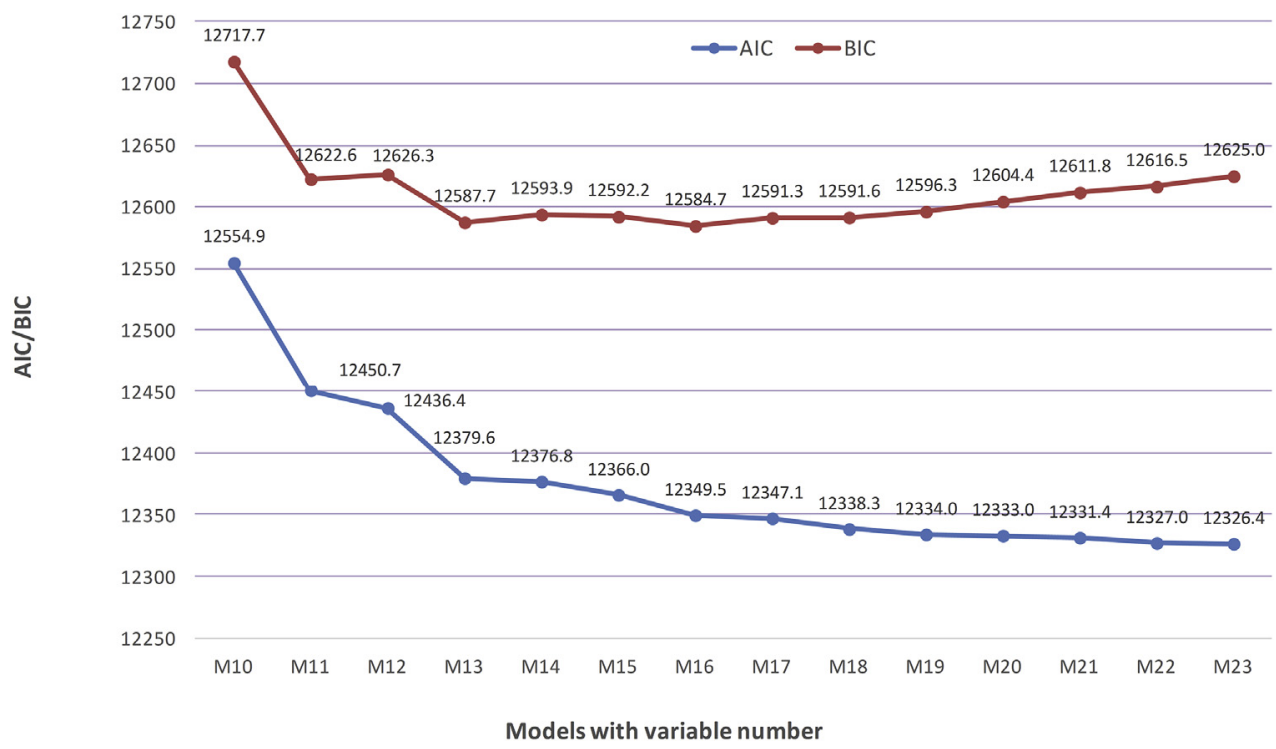

FIGURE 2. AIC and BIC gradient with increasing number of variables based on bootstrap coverage increment. AIC, Akaike information criteria; $B I C$, Bayesian information criteria. 

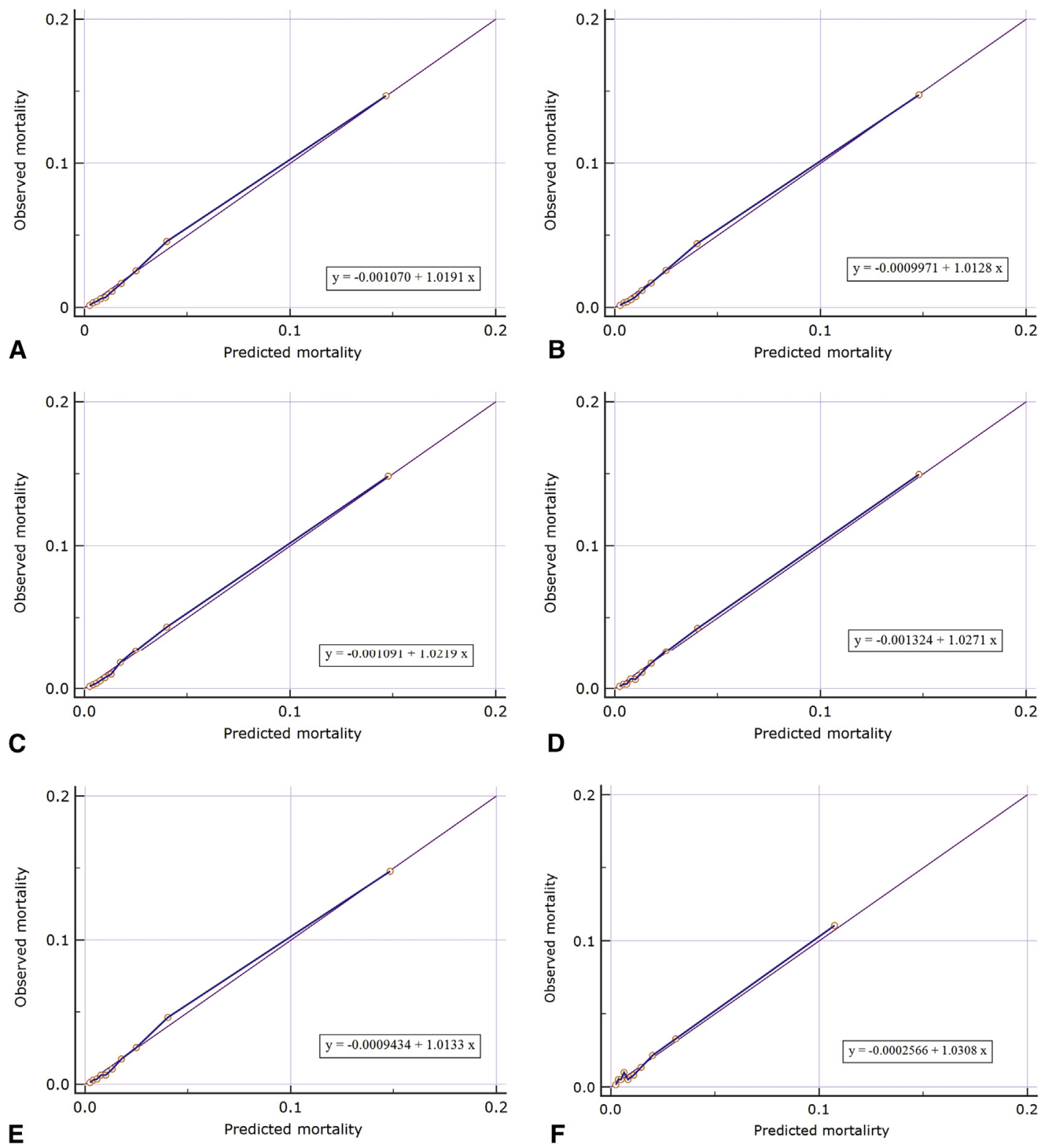

FIGURE 3. Decile-decile plots of bootstrap and automated models and validation model. A, Bootstrap ROC model; B, bootstrap BIC model; C, bootstrap AIC model; D, automated model; E, all-variable model; and F, bootstrap ROC model in validation data.

model, especially for low-risk patients (Figure 4). The model also showed very good discrimination in a multifold (10) validation (AUC, $0.8384 ; 95 \% \mathrm{CI}, 0.8356-0.8413$ ) in creation data as well as in external validation (AUC, 0.8156; 95\% CI, 0.7936-0.8375) (Table E5).

\section{DISCUSSION}

This study is the first demonstration in the surgical literature that compares different statistical approaches for developing a parsimonious risk prediction model. A risk prediction model needs to maintain a balance between the number of variables in the model and predictive accuracy. ${ }^{26}$
It is important to have a model without variables that may add little or no useful information. Omitting important prognostic factors has the potential to result in a biased estimation of the regression coefficients and inaccurate prediction. ${ }^{27}$ Hence, the trade-off between parsimony and prediction performance is a major challenge in risk prediction modeling. The inclusion of a predictor in the model should be judged against the amount of prediction power it adds to the model and one should refrain from including the new predictor if the gain seems negligible. ${ }^{5}$ When a complicated model with many predictors in practice is applied, many patients will likely need to be excluded on the basis of missing 
TABLE 2. Comparison of clinical suitability (parsimony and prediction performance) of bootstrap, automated and all predictor models

\begin{tabular}{|c|c|c|c|c|c|c|}
\hline Plausible variables & All variables & Backward elimination & Forward selection & Bootstrap AIC & Bootstrap BIC & Bootstrap ROC \\
\hline Age & $\sqrt{ }$ & $\sqrt{ }$ & $\sqrt{ }$ & $\sqrt{ }$ & $\sqrt{ }$ & $\sqrt{ }$ \\
\hline Sex & $\sqrt{ }$ & $\sqrt{ }$ & $\sqrt{ }$ & $\sqrt{ }$ & $\sqrt{ }$ & $\sqrt{ }$ \\
\hline Previous cardiac surgery & $\sqrt{ }$ & $\sqrt{ }$ & $\sqrt{ }$ & $\sqrt{ }$ & $\sqrt{ }$ & $\sqrt{ }$ \\
\hline Peripheral vascular disease & $\sqrt{ }$ & $\sqrt{ }$ & $\sqrt{ }$ & $\sqrt{ }$ & $\sqrt{ }$ & $\sqrt{ }$ \\
\hline Stroke & $\sqrt{ }$ & $\sqrt{ }$ & $\sqrt{ }$ & $\sqrt{ }$ & $\sqrt{ }$ & $\sqrt{ }$ \\
\hline Glomerular filtration rate & $\sqrt{ }$ & $\sqrt{ }$ & $\sqrt{ }$ & $\sqrt{ }$ & $\sqrt{ }$ & $\sqrt{ }$ \\
\hline Infective endocarditis & $\sqrt{ }$ & $\sqrt{ }$ & $\sqrt{ }$ & $\sqrt{ }$ & $\sqrt{ }$ & $\sqrt{ }$ \\
\hline Urgency of procedure & $\sqrt{ }$ & $\sqrt{ }$ & $\sqrt{ }$ & $\sqrt{ }$ & $\sqrt{ }$ & $\sqrt{ }$ \\
\hline Ejection fraction & $\sqrt{ }$ & $\sqrt{ }$ & $\sqrt{ }$ & $\sqrt{ }$ & $\sqrt{ }$ & $\sqrt{ }$ \\
\hline Type of procedure & $\sqrt{ }$ & $\sqrt{ }$ & $\sqrt{ }$ & $\sqrt{ }$ & $\sqrt{ }$ & $\sqrt{ }$ \\
\hline Inotropic medication use & $\sqrt{ }$ & $\sqrt{ }$ & $\sqrt{ }$ & $\sqrt{ }$ & $\sqrt{ }$ & $\sqrt{ }$ \\
\hline Angina & $\sqrt{ }$ & $\sqrt{ }$ & $\sqrt{ }$ & $\sqrt{ }$ & $\sqrt{ }$ & $\sqrt{ }$ \\
\hline NYHA class & $\sqrt{ }$ & & & $\sqrt{ }$ & $\sqrt{ }$ & $\sqrt{ }$ \\
\hline Dialysis & $\sqrt{ }$ & & & $\sqrt{ }$ & $\sqrt{ }$ & \\
\hline Congestive heart failure & $\sqrt{ }$ & $\sqrt{ }$ & $\sqrt{ }$ & $\sqrt{ }$ & $\sqrt{ }$ & \\
\hline Shock & $\sqrt{ }$ & $\sqrt{ }$ & $\sqrt{ }$ & $\sqrt{ }$ & $\sqrt{ }$ & \\
\hline Myocardial infarction & $\sqrt{ }$ & $\sqrt{ }$ & $\sqrt{ }$ & $\sqrt{ }$ & & \\
\hline Resuscitation & $\sqrt{ }$ & & & $\sqrt{ }$ & & \\
\hline Arrhythmia & $\sqrt{ }$ & $\sqrt{ }$ & $\sqrt{ }$ & $\sqrt{ }$ & & \\
\hline Left main disease $<50 \%$ & $\sqrt{ }$ & $\sqrt{ }$ & $\sqrt{ }$ & $\sqrt{ }$ & & \\
\hline Hypertension & $\sqrt{ }$ & & & $\sqrt{ }$ & & \\
\hline Respiratory disease & $\sqrt{ }$ & $\sqrt{ }$ & $\sqrt{ }$ & $\sqrt{ }$ & & \\
\hline Hypercholesterolemia & $\sqrt{ }$ & & & $\sqrt{ }$ & & \\
\hline Steroid use & $\sqrt{ }$ & & & & & \\
\hline Cerebrovascular disease & $\sqrt{ }$ & & & & & \\
\hline Diabetes & $\sqrt{ }$ & & & & & \\
\hline Pacemaker in situ & $\sqrt{ }$ & & & & & \\
\hline Body mass index & $\sqrt{ }$ & $\sqrt{ }$ & $\sqrt{ }$ & & & \\
\hline Number of disease vessel & $\sqrt{ }$ & $\sqrt{ }$ & $\sqrt{ }$ & & & \\
\hline Family history of heart disease & $\sqrt{ }$ & & & & & \\
\hline Immunosuppressant use & $\sqrt{ }$ & & & & & \\
\hline Intravenous nitrate use & $\sqrt{ }$ & & & & & \\
\hline Anticoagulant within $7 \mathrm{~d}$ & $\sqrt{ }$ & & & & & \\
\hline Number of variables & 33 & 21 & 21 & 23 & 16 & 13 \\
\hline AUC & 0.8504 & 0.8490 & 0.8490 & 0.8491 & 0.8470 & 0.8450 \\
\hline
\end{tabular}

$A I C$, Akaike information criteria; $B I C$, Bayesian information criteria; $R O C$, receiver operating characteristic; $N Y H A$, New York Heart Association; $A U C$, area under the ROC curve.

predictors. Furthermore, if many variables are included in a model, the likelihood of co-linearity increases which may result in biased or unstable estimation and model uncertainty. ${ }^{28}$

In this study, models generated by the use of automated variable selection approaches did not show better parsimony in comparison with bootstrap ROC model. The variable selection for risk prediction modeling should consider the clinical plausibility rather than solely relying on statistical variable selection methods. ${ }^{4}$ Automated model selection processes act as a black box, which can result in blind selection and hence using this method, a researcher possesses less control over what predictors are included and what is being eliminated. ${ }^{29}$ The use of automated variable selection methods sometimes may even produce nonreproducible regression models. Furthermore, automated selection has also the potential to create bias in the estimated regression coefficients. ${ }^{30}$ Particularly when the prevalence of events is low, the variable selection may be unstable, the estimated regression coefficients are too extreme, and the performance of the selected model is overestimated. ${ }^{7}$ Automated modelbuilding methods also can mask multicollinearity. In such models, the number of noise (unimportant) variables increases with an increase in the number of plausible predicators, which translates into a decrease in the probability of correctly identifying true predictor variables. ${ }^{27} \mathrm{~A}$ study on simulated data demonstrated that automated model selection may result in unstable models and may select noise variables. ${ }^{9}$ In the present study, the automated selection method chooses the variables body mass index and number of diseased vessel which had very poor bootstrap coverage $(21.2 \%)$ and $(14.1 \%)$, respectively (Table 2). There are potential that variable selection method 


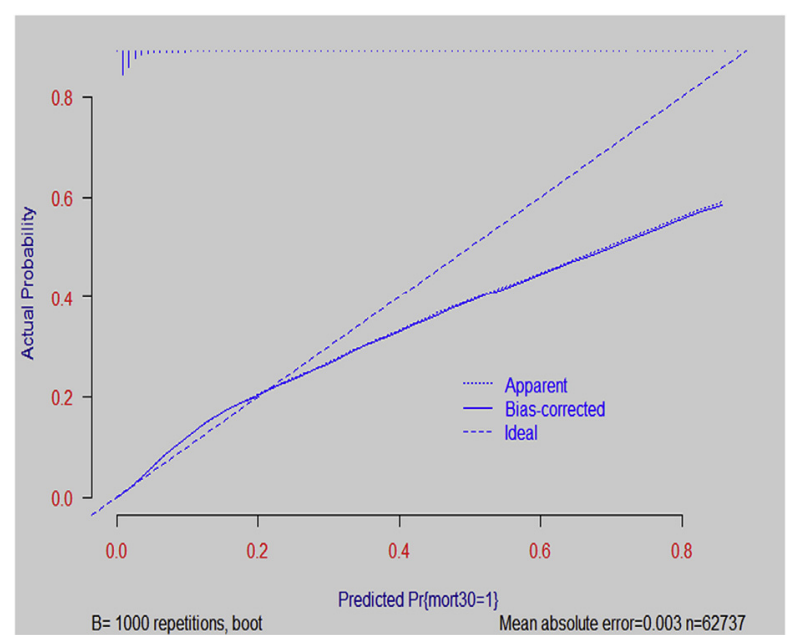

FIGURE 4. Overfitting-corrected loess nonparametric calibration curve demonstration calibration of the model.

may eliminate variables those are historically important. For instance, bootstrap ROC model didn't include variables like "cardiogenic shock," "myocardial infarction," and "angina." The model building process actually identifies the best predictors, among the set of similar predictors, which explains most variation in the outcome. In this process, it may eliminate a variable that is historically important; however, the model keeps a surrogate that explains the variation from the excluded variable. It could be that inclusion either or all of "New York Heart Association class," "urgency of operation," and "ejection fraction" is enough to capture the variation of those eliminated variables. For example, the model with 16 and 18 variables included "'cardiogenic shock" and "myocardial infarction" but didn't perform better than the models without these variables.

To minimize the limitation of automated model selection methods, backward elimination in combination with bootstrap resampling was proposed by Austin and $\mathrm{Tu}^{31}$; however, this approach did not improve the ability of variable selection to identify the true predictors of an outcome. ${ }^{32}$ Reasons why this combination method did not work could be that the automated model selection was repeated in all 1000 bootstrap samples, and hence the limitation of the automated variable selection persisted. To overcome this limitation, in this study, within each bootstrap sample the multivariable logistic regression was run independently of automated method. A similar approach was used to develop several models for predicting 30-day mortality in the ANZSCTS database. ${ }^{3,16,33}$ In this approach, the researcher has the freedom to decide on inclusion and exclusion of predictors for the final model based on theoretical and clinical plausibility and parsimony.

Small degrees of random variation in one dataset can have a substantial influence on the variables that are identified as independent predictors. Thus, it is likely that no one regression model estimated on one dataset can conclusively identify the independent predictors. ${ }^{11}$ Bootstrap resampling has been considered as a solution for the sampling variation. The bootstrap method can provide insight in the distribution of a summary measure from a sample. The bootstrap method draws samples from the original sample to introduce a random element. ${ }^{12,34}$

This study showed that bootstrap ROC method generates a parsimonious risk prediction. The model performed very well in the validation dataset. With only 13 predictors, this model showed a competitive discrimination power (AUC: 0.8156) compared with other commonly used risk prediction models such as The Society of Thoracic Surgeons (AUC: $0.8120,31$ predictors) ${ }^{1}$ and European System for Cardiac Operative Risk Evaluation II (AUC: $0.8095,18$ predictors). ${ }^{35}$

The bootstrap ROC method also can be used for developing parsimonious risk prediction model for other postoperative outcomes such as new renal failure, stroke, postoperative atrial fibrillation, pneumonia, and long-term mortality in cardiac surgery. This method also could be a useful tool for risk prediction modeling in other disciplines of research.

One limitation of this research was that all the competing models were developed for all procedures and not for a specific procedure type. It is generally hypothesized that procedure specific models performs better than the global model. Furthermore, many of the popularly used models in the field are for general cardiac surgery. The objective of this study, however, was to compare the model development methods. All the models were developed with the same process; hence, the findings in this study were not affected by case-mix. Another limitation of this study was that a number of other methods are available for screening and identification of potential candidate variables including random forest classification, classification and regressing tree (CART) etc. In this study, however, we only compared the methods currently popularly being used in the field of risk prediction modeling.

In conclusion, clinical suitability in terms of parsimony and prediction performance can be achieved by the use of bootstrap resampling in conjunction with ROC for the development of risk prediction models. We recommend this method for future risk prediction model development.

\section{Conflict of Interest Statement}

Authors have nothing to disclose with regard to commercial support.

The Australian and New Zealand Society of Cardiac and Thoracic Surgeons (ANZSCTS) National Cardiac Surgery Database Program is funded by the Department of Health, Victoria, the Health Administration Corporation (GMCT) and the Clinical Excellence Commission (CEC), NSW, and funding 
from individual Units. ANZSCTS Research activities are supported through a National Health and Medical Research Council Senior Research Fellowship and Program Grant awarded to C.M. Reid. M.N. Karim has an Australian Postgraduate Awards Scholarship from Monash University. The funding bodies had no role in the design and conduct of the study, the collection, management, analysis and interpretation of the data, or the preparation, review, or approval of the manuscript.

\section{References}

1. Shahian DM, O'Brien SM, Filardo G, Ferraris VA, Haan CK, Rich JB, et al. The Society of Thoracic Surgeons 2008 cardiac surgery risk models: part 1-coronary artery bypass grafting surgery. Ann Thorac Surg. 2009; 89(suppl 1):S2-22.

2. Nashef SA, Roques F, Michel P, Gauducheau E, Lemeshow S, Salamon R, et al. European system for cardiac operative risk evaluation (EuroSCORE). Eur $J$ Cardiothorac Surg. 1999;16:9-13.

3. Reid C, Billah B, Dinh D, Smith J, Skillington P, Yii M, et al. An Australian risk prediction model for 30-day mortality after isolated coronary artery bypass: the AusSCORE. J Thorac Cardiovasc Surg. 2009;138:904-10.

4. Steyerberg EW. Clinical Prediction Models: A Practical Approach to Development, Validation, and Updating. New York: Springer Science; 2009.

5. Kuppermann N, Willits N. In response to "Statistical Models and Occam's Razor." Acad Emerg Med. 2000;7:100-3.

6. Steyerberg EW, Vergouwe Y. Towards better clinical prediction models: seven steps for development and an ABCD for validation. Eur Heart J. 2014;35:1925-31.

7. Harrell FE Jr, Lee KL, Califf RM, Pryor DB, Rosati RA. Regression modelling strategies for improved prognostic prediction. Stat Med. 1984;3:143-52.

8. Miller A. Subset Selection in Regression. 2nd ed. Boca Raton, FL: Chapman \& Hall/CRC; 2002.

9. Austin PC, Tu JV. Automated variable selection methods for logistic regression produced unstable models for predicting acute myocardial infarction mortality. $J$ Clin Epidemiol. 2004;57:1138e46.

10. Manor O, Segal E. Predicting disease risk using bootstrap ranking and classification algorithms. PLOS Comput Biol. 2013;9:e1003200.

11. Austin PC. Using bootstrap to improve estimation and confidence intervals for regression coefficients selected using backward variable elimination. Stats Med. 2008;27:3286-300.

12. Breiman L. Bagging predictors. Machine Learning. 1996;24:123-40.

13. Akaike H. Statistical predictor identification. Ann Inst Math Stat. 1970;22: 203-17.

14. Schwarz GE. Estimating the dimension of a model. Ann Stat. 1978;6:461-4.

15. Billah B, Huq MM, Smith JA, Sufi F, Tran L, Shardey GC, et al. AusSCORE II in predicting 30-day mortality after isolated coronary artery bypass grafting in Australia and New Zealand. J Thorac Cardiovasc Surg. 2014;148:1850-5.e2.

16. Billah B, Reid CM, Shardey GC, Smith JA. A preoperative risk prediction model for 30-day mortality following cardiac surgery in an Australian cohort. Eur J Cardiothorac Surg. 2010;37:1086-92.
17. Maximus S, Milliken JC, Danielsen B, Khan J, Shemin R, Carey JS. Defining operative mortality: impact on outcome reporting. J Thorac Cardiovasc Surg. 2016;151:1101-10.

18. van Buuren S, Boshuizen HC, Knook DL. Multiple imputation of missing blood pressure covariates in survival analysis. Stat Med. 1999;18:681-94.

19. Rubin DB. Multiple Imputation for Nonresponse in Surveys. Hoboken, NJ: John Wiley \& Sons; 1987.

20. StataCorp. Stata Statistical Software, Release 14. College Station, TX: Statacorp LP; 2015.

21. Miyata H, Motomura N, Yozu R, Kyo S, Takemoto S. Cardiovascular surgery risk prediction from the patient's perspective. J Thorac Cardiovasc Surg. 2011;142: e71-6.

22. Royston P, Ambler G, Sauerbrei W. The use of fractional polynomials to model continuous risk variables in epidemiology. Int J Epidemiol. 1999;28: 964-74.

23. Sauerbrei W, Royston P. Building multivariable prognostic and diagnostic models: transformation of the predictors by using fractional polynomials. $J$ Roy Stat Soc Ser A. 1999;162:71-94.

24. Wood AM, White IR, Royston P. How should variable selection be performed with multiply imputed data? Stat Med. 2008;27:3227-46.

25. Sauerbrei W, Schumacher M. A bootstrap re-sampling procedure for mode building: application to the Cox regression model. Stat Med. 1992;11: 2093-109.

26. Wears RL, Lewis RJ. Statistical models and Occam's razor. Acad Emerg Med. 1999;6:93-4.

27. Murtaugh PA. Methods of variable selection in regression modelling. Commun Stat Simulation Computation. 1998;27:711-34

28. Greenland S. Modelling and variable selection in epidemiologic analysis. Am J Public Health. 1989;79:340-9.

29. Derkson S, Keselman HJ. Backward, forward and stepwise automated subse selection algorithms: frequency of obtaining authentic and noise variables. Br J Math Stat Psychol. 1992;45:265-82.

30. Hurvich CM, Tsai CL. The impact of model selection on inference in linear regression. Am Stat. 1990;44:214-7.

31. Austin PC, Tu JV. Bootstrap methods for developing predictive models. Am Stat. 2004;58:131-7.

32. Austin PC. Bootstrap model selection had similar performance for selecting authentic and noise variables compared to backward variable elimination: a simulation study. J Clin Epidemiol. 2008;61:1009-17.e1.

33. Ariyaratne TV, Billah B, Yap C-H, Dinh D, Smith JA, Shardey GC, et al. An Australian risk prediction model for determining early mortality following aortic valve replacement. Eur J Cardiothorac Surg. 2011;39:815-21.

34. Efron B, Tibshirani R. An Introduction to the Bootstrap. New York: Chapman \& Hall; 1993.

35. Nashef SAM, Roques F, Sharples LD, Nilsson J, Smith C, Goldstone AR, et al EuroSCORE II. Eur J Cardiothorac Surg. 2012;41:734-45.

Key Words: bootstrap resampling, automated model, risk prediction model, cardiac surgery 
TABLE E1. ANZSCTS registry variable information

\section{Variables}

$1 \quad$ Preoperative (101)

Patient demography

Patient risk factor

Preoperative cardiac status

Previous intervention

Hemodynamic state

2

Intra- operative (114)

Operative status

Minimally invasive

CPB support

Coronary bypass

Valve surgery

$3 \quad$ Postoperative (72)

Postoperative support

Complication

Mortality/readmission

Total

$C P B$, Cardiopulmonary bypass.

Data field number
24

19

33

16

09

41

04

17

12

40

15

36

21

287
TABLE E2. Number ( $\%$ ) of missing observations for predictors in the development dataset

\begin{tabular}{lrl}
\hline & \multicolumn{2}{c}{ Missing values } \\
\cline { 2 - 3 } \multicolumn{1}{c}{ Plausible variables } & $\mathbf{n}$ & $\%$ \\
\hline Age & 13 & 0 \\
Sex & 0 & 0 \\
Peripheral vascular disease & 215 & 0.34 \\
Stroke & 0 & 0 \\
Glomerular filtration rate & 576 & 0.91 \\
Infective endocarditis & 207 & 0.33 \\
Urgency of procedure & 37 & 0.06 \\
Ejection fraction & 1951 & 3.07 \\
Inotropic medication use & 207 & 0.33 \\
Type of procedure & 786 & 1.24 \\
Previous cardiac surgery & 182 & 0.29 \\
NYHA class & 3355 & 5.28 \\
Angina & 581 & 0.91 \\
Shock & 199 & 0.31 \\
Congestive heart failure & 195 & 0.31 \\
Myocardial infarction & 172 & 0.27 \\
Resuscitation & 201 & 0.32 \\
Arrhythmia & 220 & 0.35 \\
Hypertension & 207 & 0.33 \\
Left main disease $<50 \%$ & 581 & 0.91 \\
Hypercholesterolemia & 217 & 0.34 \\
Respiratory disease & 195 & 0.31 \\
Steroid use & 211 & 0.33 \\
Diabetes & 327 & 0.52 \\
Pacemaker in situ & 429 & 0.68 \\
Body mass index & 339 & 0.53 \\
Number of disease vessel & 703 & 1.11 \\
Immunosuppressant use & 206 & 0.32 \\
Intravenous nitrate use & 211 & 0.33 \\
Anticoagulant use & 221 & 0.35 \\
Mortality in 30 d & 280 & 0.44 \\
\hline NYHA New York Heart Association & & \\
\hline & &
\end{tabular}

NYHA, New York Heart Association. 
TABLE E3. Descriptive statistic s of plausible categorical variables

Variables
Sex
Male
Female
Family history of heart disease

No

Yes

Frequency

Percent

Previous cardiac surgery

$$
\text { No }
$$

Yes

Peripheral vascular disease

$$
\begin{aligned}
& \text { No } \\
& \text { Yes }
\end{aligned}
$$

Respiratory disease

$$
\text { No }
$$

Yes

Cerebrovascular disease

No

Yes

Stroke

$$
\text { No }
$$

Yes

Hypertension

No

Yes

Diabetes

$$
\begin{aligned}
& \text { No DM } \\
& \mathrm{DM}+\text { no insulin } \\
& \mathrm{DM}+\text { insulin }
\end{aligned}
$$

Hypercholesterolemia

$$
\begin{aligned}
& \text { No } \\
& \text { Yes }
\end{aligned}
$$

IE

No IE

IE treated

IE active

Left main disease

No

Yes

Number of diseased vessels

\section{None}

One

Two

Three

NYHA class

$\begin{array}{lr}\text { I, II } & 42,367 \\ \text { III } & 13,599 \\ \text { IV } & 4202\end{array}$

Cardiogenic shock

$\begin{array}{lr}\text { No } & 61,820 \\ \text { Yes } & 1504\end{array}$

Resuscitation

\begin{tabular}{lrc} 
No & 62,559 & 98.48 \\
Yes & 763 & 1.2 \\
\hline
\end{tabular}

72.18

27.82

60.58

30.41

91.18

8.54

89.3

10.37

85.89

13.81

88.34

11.33

97.91

2.09

27.19

72.48

71.28

21.16

7.14

32.04

67.62

97.22

0.87

1.59

81.33

17.75

24.99

\begin{tabular}{|c|c|c|}
\hline Variables & Frequency & Percent \\
\hline \multicolumn{3}{|l|}{ Congestive heart failure } \\
\hline Yes & 47,953 & 75.49 \\
\hline No & 15,375 & 24.2 \\
\hline \multicolumn{3}{|l|}{ Ejection fraction } \\
\hline Normal, $>60 \%$ & 31,391 & 49.42 \\
\hline Mild, $46 \%-60 \%$ & 18,321 & 28.84 \\
\hline Moderate, $31 \%-45 \%$ & 8689 & 13.68 \\
\hline Severe, $\leq 30$ & 3171 & 4.99 \\
\hline \multicolumn{3}{|l|}{ Arrhythmia } \\
\hline No & 53,078 & 83.56 \\
\hline Yes & 10,225 & 16.1 \\
\hline \multicolumn{3}{|l|}{ Pacemaker in situ } \\
\hline No & 61,890 & 97.43 \\
\hline Yes & 1204 & 1.9 \\
\hline \multicolumn{3}{|l|}{ Angina } \\
\hline No angina & 21,225 & 33.41 \\
\hline Stable angina & 30,664 & 48.27 \\
\hline Unstable angina & 11,053 & 17.4 \\
\hline \multicolumn{3}{|l|}{ Previous MI } \\
\hline No & 38,817 & 61.11 \\
\hline Yes & 24,534 & 38.62 \\
\hline \multicolumn{3}{|l|}{ Inotrope use } \\
\hline No & 61,630 & 97.02 \\
\hline Yes & 1686 & 2.65 \\
\hline \multicolumn{3}{|l|}{ Intravenous nitrates } \\
\hline No & 60,078 & 94.58 \\
\hline Yes & 3234 & 5.09 \\
\hline \multicolumn{3}{|l|}{ Anticoagulant use } \\
\hline No & 50,973 & 80.24 \\
\hline Yes & 12,329 & 19.41 \\
\hline \multicolumn{3}{|l|}{ Steroids use } \\
\hline No & 61,806 & 97.3 \\
\hline Yes & 1506 & 2.37 \\
\hline \multicolumn{3}{|l|}{ Immunosuppressive use } \\
\hline No & 61,784 & 97.26 \\
\hline Yes & 1533 & 2.41 \\
\hline \multicolumn{3}{|l|}{ Procedure type } \\
\hline Isolated CABG & 36,085 & 56.81 \\
\hline Valve(s) only & 10,489 & 16.51 \\
\hline Valve(s) + CABG & 6746 & 10.62 \\
\hline Other & 9417 & 14.82 \\
\hline \multicolumn{3}{|l|}{ Urgency of operation } \\
\hline Elective & 41,829 & 65.85 \\
\hline Urgent & 18,478 & 29.09 \\
\hline Emergency & 2924 & 4.6 \\
\hline Salvage & 255 & 0.4 \\
\hline \multicolumn{3}{|l|}{ 30-d mortality } \\
\hline No & 61,540 & 97.31 \\
\hline Yes & 1703 & 2.69 \\
\hline
\end{tabular}

8.29

17.86

47.75

66.7

21.41

6.61

97.32

2.37
51,664
TABLE E3. Continued

Association; $M I$, myocardial infarction; $C A B G$, coronary artery bypass grafting. 
TABLE E4. Descriptive statistic s of plausible numerical variables

\begin{tabular}{lccr}
\hline \multicolumn{1}{c}{ Predictors } & n & Mean & \multicolumn{1}{c}{ SD } \\
\hline Age, y & 63,510 & 65.67 & 12.83 \\
Body mass index & 63,351 & 28.48 & 9.74 \\
Estimated glomerular filtration rate & 62,947 & 82.15 & 37.10 \\
\hline
\end{tabular}

$S D$, Standard deviation.
TABLE E5. Bootstrap ROC model

\begin{tabular}{|c|c|c|}
\hline Predictors & OR $(95 \%$ CI $)$ & $P$ valu \\
\hline Age & $1.024(1.018-1.029)$ & $<.001$ \\
\hline Sex (female) & $1.414(1.265-1.581)$ & $<.001$ \\
\hline eGFR & $0.990(0.987-0.992)$ & $<.001$ \\
\hline \multicolumn{3}{|l|}{ Infective endocarditis (ref: none) } \\
\hline Treated endocarditis & $1.205(0.745-1.948)$ & .446 \\
\hline Active endocarditis & $2.437(1.874-3.169)$ & $<.001$ \\
\hline \multicolumn{3}{|l|}{ Urgency (ref: elective) } \\
\hline Urgent & $1.567(1.371-1.791)$ & $<.001$ \\
\hline Emergency/salvage & $4.611(3.884-5.475)$ & $<.001$ \\
\hline \multicolumn{3}{|l|}{ Ejection fraction (ref: $>60 \%$ ) } \\
\hline Mild $(46 \%-60 \%)$ & $1.242(1.081-1.427)$ & .002 \\
\hline Moderate $(31 \%-45 \%)$ & $1.634(1.397-1.910)$ & $<.001$ \\
\hline Severe $(\leq 30 \%)$ & $2.346(1.895-2.904)$ & $<.001$ \\
\hline \multicolumn{3}{|l|}{ NYHA class (ref: I and II) } \\
\hline III & $1.467(1.279-1.681)$ & $<.001$ \\
\hline IV & $1.725(1.463-2.032)$ & $<.001$ \\
\hline \multicolumn{3}{|l|}{ Procedure type (ref: CABG) } \\
\hline Valve & $1.462(1.209-1.767)$ & .001 \\
\hline $\mathrm{CABG}+$ valve & $2.043(1.738-2.402)$ & $<.001$ \\
\hline Others & $2.968(2.546-3.460)$ & $<.001$ \\
\hline Peripheral vascular disease & $1.617(1.406-1.859)$ & $<.001$ \\
\hline Stroke & $5.865(4.985-6.901)$ & $<.001$ \\
\hline Inotrope administration & $2.293(1.916-2.743)$ & $<.001$ \\
\hline \multicolumn{3}{|l|}{ Angina } \\
\hline Stable & $0.930(0.810-1.066)$ & .297 \\
\hline Unstable & $1.260(1.066-1.489)$ & .007 \\
\hline Previous cardiac surgery & $1.687(1.459-1.951)$ & $<.001$ \\
\hline
\end{tabular}
$N Y H A$, New York Heart Association; $C A B G$, coronary artery bypass grafting. 